\title{
Philosophiques
}

\section{Quel développement et pour qui?}

\section{Joseph Pestieau}

Volume 16, numéro 2, automne 1989

URI : https://id.erudit.org/iderudit/027083ar

DOI : https://doi.org/10.7202/027083ar

Aller au sommaire du numéro

\section{Éditeur(s)}

Société de philosophie du Québec

ISSN

0316-2923 (imprimé)

1492-1391 (numérique)

Découvrir la revue

\section{Citer ce document}

Pestieau, J. (1989). Quel développement et pour qui? Philosophiques, 16(2), 327-346. https://doi.org/10.7202/027083ar

\section{Résumé de l'article}

My paper is divided into two parts. In the first one, I try to categorize the different strands in the criticism of development. In the second part, I discuss the values which could give a sound direction to development.I distinguish five different kinds of criticism.- First, the Marxist inspired approach. Development is seen as the result of imperialism, the satellization of a periphery around and for the benefit of a center or a class in a center.- Second, the criticism of the extreme left. According to it, development no longer has either subjects or ends. The anonymous industrial system feeds on itself.- Third, the Greens' criticism. Ecological considerations are becoming more and more intractable limits to development.- A fourth kind of criticism is the culturalist one : development tends to suppress the originalities of the world's different cultures.- Fifth, some critics reject the technical mastery of the universe and seek a more convivial and more receptive attitude. The two last criticisms are still more radical. But we cannot turn the clock back, our only choice is to limit and repair the damage development has already done. We must redirect it, not refuse it. In the second part of my paper, I indicate the direction develop- ment should take : an utilitarian direction, that is to say, a maximization of collective utilities within a plan. But I stress three shortcomings of such an approach which need to be corrected by entrenched funda- mental rights.- First, utilitarianism aims at collective benefit but can be blind to the distribution of costs and to the rights of persons and minorities.- Second, planning inevitably ignores those ends whose beneficial consequences cannot be factored in. For example, the health of the elderly is a dead end whereas the health of the labour force of the future is desirable for the stream of benefits it promises.Third, our values change, so planning has to be a process of constant revision. A democratic process, founded on and protected by fundamental rights can counter these three shortcomings.- First, defending each person's rights is a way to limit sacrifices imposed on individuals in the name of a collective good or development.- Second, rights of association and political expression for the poorest ensure that they have a say in choosing social policies.- Third, the respect of each person's fundamental rights is an ultimate end and gives a sense of direction to all social policies.
Ce document est protégé par la loi sur le droit d'auteur. L’utilisation des services d’Érudit (y compris la reproduction) est assujettie à sa politique d'utilisation que vous pouvez consulter en ligne.

https://apropos.erudit.org/fr/usagers/politique-dutilisation/ 
PHILOSOPHIQUES, Vol. XVI, Numéro 2, Automne 1989

\title{
INTERVENTIONS
}

\section{QUEL DÉVELOPPEMENT ET POUR QUI ? *}

\author{
par Joseph Pestieau
}

ABSTRACT My paper is divided into two parts. In the first one, I try to categorize the different strands in the criticism of development. In the second part, I discuss the values which could give a sound direction to development.

I distinguish five different kinds of criticism.

- First, the Marxist inspired approach. Development is seen as the result of imperialism, the satellization of a periphery around and for the benefit of a center or a class in a center.

- Second, the criticism of the extreme left. According to it, development no longer has either subjects or ends. The anonymous industrial system feeds on itself.

- Third, the Greens' criticism. Ecological considerations are becoming more and more intractable limits to development.

- A fourth kind of criticism is the culturalist one : development tends to suppress the originalities of the world's different cultures.

- Fifth, some critics reject the technical mastery of the universe and seek a more convivial and more receptive attitude.

The two last criticisms are still more radical. But we cannot turn the clock back, our only choice is to limit and repair the damage development has already done. We must redirect it, not refuse it.

In the second part of my paper, I indicate the direction development should take : an utilitarian direction, that is to say, a maximization of collective utilities within a plan. But I stress three shortcomings of such an approach which need to be corrected by entrenched fundamental rights.

* Ce rexte reprend et incègre la communication de l'auteur présentée à Windsor, le 25 mai 1988, au Congrès de l'Association Canadienne de Philosophie, et un séminaire présenté à Ottawa, le 6 novembre 1987, au «Centre de recherches pour le développement international". 
- First, utilitarianism aims at collective benefit but can be blind to the distribution of costs and to the rights of persons and minorities.

- Second, planning inevitably ignores those ends whose beneficial consequences cannot be factored in. For example, the health of the elderly is a dead end whereas the health of the labour force of the future is desirable for the stream of benefits it promises.

- Third, our values change, so planning has to be a process of constant revision.

A democratic process, founded on and protected by fundamental rights can counter these three shortcomings.

- First, defending each person's rights is a way to limit sacrifices imposed on individuals in the name of a collective good or development.

- Second, rights of association and political expression for the poorest ensure that they have a say in choosing social policies.

- Third, the respect of each person's fundamental rights is an ultimate end and gives a sense of direction to all social policies.

\section{INTRODUCTION}

Développement économique et social, sous-développement, aide internationale, ces termes mêmes ont été critiqués : ils seraient irrémédiablement grevés par l'idéologie impérialiste de l'Occident, il vaudrait mieux laisser les diverses cultures à leur singularité, elles s'appauvrissent en participant au commerce international, elles perdent leur âme en s'enrichissant, les inégalités et les dépendances s'accroissent avec l'industrialisation. Je tâcherai de mettre un peu d'ordre dans ce concert de critiques. Je tâcherai ensuite de dégager des repères qui devraient orienter un développement véritable. Dans ce contexte, je parlerai du respect des droits de la personne comme correctif à une perspective utilitariste.

\section{CRITIQUES DU DÉVELOPPEMENT}

Pour les besoins de la cause, je distinguerai cinq types de critiques : le premier dénonce l'impérialisme culturel de l'Occident, le second parle du non-sens et de la boulimie de l'industrialisation, le troisième porte sur les limites écologiques de la croissance économique. Les quatrième et cinquième vont plus loin: ils 
paraissent refuser le développement économique lui-même et non seulement ses excès. Certes, un auteur tel qu' Yvan Illich pourrait facilement prendre place dans chacun des cinq types. C'est que ceux-ci se recoupent l'un l'autre. Mais, pour la clarté de l'exposé, je les maintiendrai séparés.

Le premier type de critiques est d'abord le fait de la gauche marxisante. Le développement, dans cette perspective, est celui du capitalisme. Il se produit dans un ou quelques centres, mais réduit le reste du monde, la périphérie, à un état de dépendance. Il détruit les cultures particulières et les équilibres économiques qu'elles connaissaient, parce qu'il a un besoin insatiable de matières premières ou de main-d'œuvre à bon compte, ou de consommateurs, notamment pour des produits qui sont déjà passés de mode au centre. En France, dès les années 50, une pensée humaniste et chrétienne a repris ce point de vue, et lui a fait largement écho dans des milieux qui ne sont pas que de gauche.

Dans son Economie du XX $X^{e}$ siècle (PUF, Paris, 1961), François Perroux, un des premiers, a tâché de comprendre comment s'articulaient les rapports entre périphérie et centre. Il a étudié le rôle des sous-centres, relais de la diffusion du progrès ou des effets pervers de l'impérialisme. Dans cette analyse, Sao Paolo était le type du sous-centre et le Nordeste, le type de la périphérie. Celso Furtado, un brésilien, a analysé Le Mythe du développement (traduction française: Anthropos, Paris, 1976) dans le TiersMonde. Dans de nombreuses publications, largement traduites, il a insisté sur la dépendance qui s'accroît, dans l'ensemble du TiersMonde, vis-à-vis des pays industrialisés. André Gunder Frank, sur un ton plus virulent, a parlé du Développement du sous-développement (Maspero, Paris, 1972). Comme Franz Fanon dans Les Damnés de la terre (Maspero, 1968, première édition en 1961), il a dénoncé les bourgeoisies colonisées, imitatrices veules des bourgeoisies colonisatrices, relais serviles de l'impérialisme. Il faut lire à ce propos son Lumpen-bourgeoisie et lumpen-développement (Maspero, 1971).

L'anthropologie, marxiste ou non, a repris le même discours, mais à une autre échelle d'observation. Elle envisage des cultures singulières de la périphérie et prend parti pour ces cultures bousculées, déséquilibrées, en crise du fait de l'irruption, en leur 
sein, de la culture occidentale et de ses modèles de développement. Elle critique le remplacement d'une économie de subsistance par des monocultures et le salariat. Elle dénonce la dépendance progressive des indigènes vis-à-vis de biens de consommation importés, la destruction de leur vie traditionnelle et de leurs valeurs, l'exploitation ou la surexploitation dont ils sont l'objet. Par exemple, dans Femmes, greniers, et capitaux (Maspero, 1975), Claude Meillassoux a montré comment l'autarcie familiale, assurée par l'horticulture et le petit élevage dont se chargent les femmes, permet aux hommes d'être des travailleurs saisonniers dont le salaire est inférieur au coût minimal de leur propre entretien et de leur propre reproduction.

Les critiques actuelles contre les prix et les subsides agricoles, en pratique aux États-Unis et dans la Communauté européenne, illustrent bien un des aspects de l'impérialisme. Les lobbies agricoles, en Europe et aux États-Unis, ont réussi à obtenir que leurs gouvernements manipulent les prix d'une série de denrées. D'où surproduction, gaspillage et dommages écologiques, effondrement des prix internationaux, dumping ou don de certaines denrées dans des pays du Tiers-Monde. Du coup, ces pays sont de plus en plus dépendants de l'étranger pour se nourrir, et leur agriculture ne peut se développer comme elle le devrait.

Ce que j'appelle l'impérialisme ne résulte pas nécessairement d'une stratégie délibérée, mais n'est pas non plus le résultat des forces aveugles du marché. Il y a des groupes qui peuvent influencer celui-ci et les gouvernements. Il y a des gouvernements qui peuvent imposer leur point de vue à d'autres gouvernements. Chacun poursuit, avec les moyens dont il dispose, des objectifs souvent immédiats. Il en résulte des effets de domination, souvent anonymes et sans grand dessein, à ras des intérêts à court terme. Pour les combattre, il convient de dénoncer les mancuvres des lobbies, la vision à court terme des acteurs économiques et des gouvernements. Il faut que les pays dominés voient clairement ce qui les maintient dans cet état, qu'ils en appellent aux opinions des pays dominants, car c'est là qu'ils trouveront des alliés politiques influents. Il faudrait surtout qu'ils aient la volonté de centrer leur développement sur leurs besoins. Et là n'est pas la moindre difficulté : ces pays ont-ils des élites qui peuvent s'identifier au peuple plutôt qu'à des phantasmes d'origine étrangère? 
Manifestement, la critique du développement que je viens d'évoquer ne porte pas sur le développement lui-même, mais sur le fait qu'il n'est pas centré sur les besoins des peuples, sur le fait qu'il est inégal, sur le fait qu'il entraîne le sous-développement chez les uns et le gaspillage chez les autres.

La seconde catégorie de critiques est celle des gauchistes. Ceux-ci n'attaquent pas seulement les politiques de développement, mais la mentalité même qui veut la croissance économique et qui, en retour, est façonnée par cette croissance. Herbert Marcuse fut sans doute leur porte-parole le plus cohérent et le plus connu. Selon Marcuse, l'homme veut le plaisir mais accepte de le remettre à plus tard, pour épargner, investir, organiser les moyens qui lui donneront plus de plaisir dans l'avenir. Dans ce procès, se crée la civilisation. Mais, avec l'industrialisation, la civilisation a mal tourné : l'homme a non seulement accru de façon extraordinaire ses moyens, il veut les accumuler sans raison. Il n'a plus d'autre fin qu'augmenter ses capacités de production et sa consommation. En fait, le phénomène n'est pas seulement psychologique. Il faut comprendre qu'un système industriel fort complexe s'est développé et, désormais, chacun est conditionné par le rôle qu'il y joue, obnubilé par les attentes que lui impose ce système, embrigadé par celui-ci pour le servir et le développer toujours davantage.

Dans mon Essai contre le défaitisme politique (Presses de l'Université de Montréal, 1973), j'ai analysé comment l'intelligence économique, la prudence du gestionnaire ou de l'épargnant, le besoin d'un emploi ou d'une promotion professionnelle chez le travailleur, en arrivent à stéréliser l'imagination politique. Jacques Ellul, dans sa Métamorphose du bourgeois (Calmann-Lévy, Paris, 1967), a insisté sur l'insertion du manager contemporain dans une organisation qu'il ne domine plus et qui lui impose une manière de faire et de penser. John Kenneth Galbraith, dans The New Industrial State (Houghton, Mifflin \& Co., Boston, 1967), a montré comment les initiatives et décisions industrielles sont le résultat d'un consensus des cadres, de ce qu'il appelle la " technostructure». Andrew Schonfield, dans Modern Capitalism (Oxford University Press, 1965), a souligné l'accord foncier entre l'État et le secteur privé, pour entretenir une certaine croissance. Tous ces auteurs, dans des perspectives très différentes, expliquent que cette croissance n'a pas besoin d'être choisie, qu'elle résulte de 
l'organisation économique et de la mentalité que conditionne cette organisation.

Le sort du Tiers-Monde est aussi une conséquence de la boulimie et de l'absence de direction du système industriel. Celui-ci se transforme, s'élargit, détermine les intérêts et les besoins. Les hommes ne peuvent plus se déprendre des rôles, des manières de penser et de coopérer qu'ils ont mises au point pour se libérer de la nécessité. Désormais ils sont prisonniers d'une nouvelle nécessité, ils n'ont plus d'autre fin que de continuer à accumuler des moyens.

Une telle critique eut beaucoup de succès auprès des intellectuels. Elle entretint l'opposition gauchiste à la gauche, favorable à la croissance, représentative des salariés de l'industrie, acceptant les règles de jeu imposées par celui-ci. Le gauchisme prétend représenter le Tiers et le Quart-Monde qui n'ont rien à gagner dans «le système » et qui sont censés le découvrir. Selon Marcuse, ceux qui souffrent le plus de l'absurdité et du non-sens du système industriel, ne peuvent plus le supporter. Longtemps ceux qui en étaient exclus, rêvaient d'y être inclus. Des femmes et des chômeurs, comme des paysans misérables, rêvaient d'un emploi de huit à cinq. Mais ce rêve a peut-être perdu ses charmes. Certes, tous ont besoin d'un revenu, mais on ose aujourd'hui envisager d'autres voies que le salariat industriel, la consommation de masse, le développement insensé qu'exigent les institutions et les intérêts établis. C'est du moins un espoir sur lequel tablent les gauchistes.

La troisième critique que j'envisage est celle des « verts ». Elle paraissait encore marginale il y a une dizaine d'années. L'étude du Club de Rome (Meadows, Meadows, Randers, Behhens, The Limits to Growth, Universe, New York, 1972) avait poussé un cri d'alarme qui ne fut pas pris au sérieux : les projections de cette étude ne se réalisant pas telles quelles, on les a discréditées. On ne voyait pas, on ne voulait pas voir, qu'elles devaient être corrigées au fur et à mesure que les variables se précisaient. Mais depuis, la situation a changé. Des courants d'opinion influents dans des pays puissants se sont sentis agressés par la pollution, notamment par les pluies acides. Ils utilisent leur influence ou leur puissance pour dénoncer les coupables et trouver des solutions à la mesure des problèmes, c'est-à-dire des solutions internationales. Il faut noter que les sociétés riches peuvent se payer le souci écologique et ont les moyens d'y donner suite. 
Le Rapport Brundtland (Our Common Future, Oxford University Press, 1987) a clarifié, pour un large public, les problèmes que soulevaient les verts. Premièrement, il a fait un tour complet de ces problèmes. Il a ainsi donné une vue plus équilibrée de la situation, une vue que l'on peut difficilement discréditer. Deuxièmement, il a montré que le sous-développement était aussi dommageable que l'industrialisation à outrance. La pauvreté entraîne une exploitation ruineuse et irrationnelle de ressources rares. Il faut donc tirer de leur misère les régions sous-développées pour préserver un certain équilibre écologique de la planète. Troisièmement, ce rapport souligne que le développement social er économique doit être conçu dans un horizon temporel très éloigné, de façon à prévoir son propre avenir, de façon à ménager les ressources pour les générations futures. Si l'on veut être juste vis-à-vis de celles-ci et si l'on veut assurer la continuité du développement, il ne faut pas seulement se soucier des contemporains. Quatrièmement, le Rapport Brundtland propose des moyens qui n'ont rien d'utopiques pour résoudre les problèmes énormes qu'il soulève. Il fait preuve d'optimisme et d'esprit pratique, plutôt que d'annoncer l'apocalypse.

Alors que certains verts condamnaient la croissance, le Rapport Brundtland nous montre la convergence entre un développement contrôlé et la conservation des ressources de la planète. Il a, de ce fait, réussi à réconcilier des perspectives opposées. Il a aussi réussi à tracer une voie claire pour une collaboration internationale.

$$
\text { * * * } *
$$

Il n'y a pas seulement des limites naturelles à la croissance, il y a aussi des limites sociales que Fred Hirsh (Social Limits to Growth, Harvard University Press, 1978) a bien illustrées. On imagine parfois que les hommes ont des besoins limités, que le développement vise à y répondre et que la seule contrainte tient à la rareté des ressources. Mais ce qui est vrai pour le besoin de pain ne l'est pas pour le besoin de se distinguer des autres, en consommant davantage ou autrement que les autres, en possédant des biens très rares ou uniques. Ce second besoin est envahissant et probablement insatiable. Il est certainement impossible de le satisfaire pour tous. 
En suivant le type d'argument d'Albert Hirschman (Shifting Involvements. Private Interest and Public Action, Princeton University Press, 1982), on pourrait parler de limites morales ou psychologiques à la croissance. La déception entraînée par la consommation de certains biens et services, l'ennui que la richesse ne pourrait dissiper, entraîneraient à terme un désintérêt pour la croissance économique.

Ce genre de réflexion m'introduit à un tout autre type de critique du développement. Il s'agit non plus de la critique de tel ou tel travers du développement, mais d'un refus de l'idée même de développement. Ce refus peut se fonder sur un choix en faveur de l'équilibre stable, qui caractérise bien des cultures "primitives", entre besoins humains reconnus et valorisés, d'une part, et ressources locales, d'autre part. Le refus peut aussi se fonder sur une acceptation sage de notre finitude, sur une ouverture poétique au monde que nieraient l'exploitation économique et la maîtrise technique, ou sur une perception du développement comme «hubris» sans limite, aboutissant fatalement à des frustrations psychologiques et à des désordres sociaux. Ces frustrations et ces désordres apparaissent sans doute bien avant les limites écologiques.

Je distinguerai une critique culturaliste et une critique que je dirais métaphysique. Je montrerai ensuite que le développement demeure une nécessité éthique, économique et diplomatique, en dépit des discours qui semblent le charger de tous les maux.

Notre culture est caractérisée par une volonté de développement alors que beaucoup d'autres, la plupart des autres sans doute, favorisent plutôt la conservation d'un équilibre. De plus, notre culture a réussi à bousculer toutes les autres. Dans bien des cas, on pourrait même parler d'ethnocide. Disons au moins ceci : l'extension tentaculaire de la civilisation industrielle a imposé au monde entier son idée du progrès et «l'échange inégal » entre produits industrialisés et produits du Tiers-Monde. Elle a entraîné la ruine des économies locales et une certaine démoralisation de tous ceux qui ne partagent pas la vision occidentale du monde.

La critique culturaliste du développement nous dit qu'il ne faut pas détruire l'ordre moral traditionnel et la sagesse des cultures que l'Occident n'a pas encore perverties, qu'il faut les 
préserver autant que possible et y trouver une inspiration pour ramener l'Occident lui-même à plus de sagesse.

L'Occident ne pervertirait pas seulement les autres. Il serait lui-même perverti. Le désordre ne serait pas seulement le prix à payer pour passer d'un équilibre traditionnel à la nouvelle culture industrielle. Celle-ci serait elle-même un désordre. À ce propos, Martin Heidegger a défendu une thèse que je voudrais présenter brièvement. C'est une thèse qui est souvent reprise, avec plus ou moins de bonheur, pour critiquer les vices de notre société et chanter les vertus d'autres sociétés, plus sages, plus simples ou plus dépouillées.

L'homme, selon Heidegger, se définit comme être-au-mondeavec-autrui. Il se constitue en constituant le monde, en étant à l'écoute des autres et du sens des choses, en poursuivant ou en corrigeant les projets des autres à propos du monde. Il en résulte un monde plus humain, portant les traces des intentions humaines. Nous sommes nés dans un monde déjà riche d'une histoire, dans un monde où il y avait des intentions à reprendre, des projets à continuer, des occasions à saisir, des possibilités à libérer. C'est en les libérant que l'homme se libère. C'est en se mettant à l'écoute des autres et des choses que l'homme advient à lui-même. Mais il peut aussi se pervertir, rendre stériles le monde et l'histoire, s'il prétend non plus écouter mais seulement manipuler, dominer et exploiter. Il s'enferme alors dans un projet unilatéral. À travers le développement de sa maîtrise technique, il croit réaliser son destin. Mais, en fait, il devient le jouet d'un destin anonyme et aveugle. Il n'est plus à l'écoute, complice intelligent des significations à déchiffrer et à libérer pour gagner sa propre liberté. Il n'est plus qu'un imbécile tout entier obsédé par la volonté de puissance, prisonnier de ses moyens militaires, industriels ou scientifiques, sourd aux raisons que lui offrent les autres, aveugle à la poésie des choses.

Toutes ces critiques du développement - qui se réduit au développement industriel - sont à la fois très justes et bien tardives. Le monde entier est déjà entraîné dans les hauts et les bas d'une économie désormais internationale. Les sagesses comme les cultures traditionnelles peuvent disparaître en une génération, du fait de la fascination des nouveaux modèles d'écolage et de carrière, 
de consommation, d'épargne et de production. Le Tiers comme le Quart-Monde se retrouvent laissés pour compte, démoralisés et méprisés, livrés à la clochardisation, soumis à la violence endémique qui accompagne la misère, violence des misérables mais aussi et surtout violence de ceux qui les réduisent à cet état.

Il n'y a qu'une seule réponse raisonnable à cette situation : un développement qui sache tirer les ressources humaines du mépris où trop souvent elles se sont retrouvées suite à une croissance sauvage. Si celle-ci a détruit des sociétés, il faut les reconstruire, utiliser les capitaux, les techniques, les savoir-faire et les hommes disponibles. Je distingue trois motifs de procéder avec urgence, trois motifs qui devraient orienter le développement. Le premier est économique. Le maintien d'un appareil de production, ne fût-ce que pour répondre aux besoins essentiels, dépend d'une gestion prudente des ressources et des moyens formidables que nous mettons en ceuvre. Un minimum de prospérité ne peut être entretenu que si l'on se soucie d'en assurer les conditions. Cela nécessite un développement contrôlé et concerté à l'échelle du monde. Le deuxième motif est diplomatique. Les déséquilibres économiques, les écarts entre riches et pauvres, sont des facteurs parmi les plus importants de la guerre et de l'insécurité. Le troisième motif est éthique. Combattre la misère, assurer la justice sont des fins qui s'imposent souvent sans discussion. En tout cas, ce sont de telles fins qui justifient le développement économique comme la recherche de la paix et de la sécurité. Mais que veut dire combattre la misère, assurer la justice quand on parle d'aide au développement du Tiers-Monde?

\section{SELON QUELLES VALEURS INTERVENIR}

Une première position tend à affirmer que les valeurs qui devraient orienter nos interventions dans les pays en voie de développement, devraient être celles des peuples concernés par nos interventions. Certes, nous sommes tous pour la vertu: en principe, nous voudrions tous pouvoir reconnaître les valeurs des peuples. Mais qu'est-ce qu'un peuple? Si on entend par là un groupe partageant les mêmes valeurs, n'y a-t-il pas autant de peuples que de tribus, que de classes sociales? D'autre part, qui parle au nom d'un peuple? Dans la plupart des pays en voie de 
développement, le peuple est sans voix, sans institutions politiques pour s'exprimer. Parfois même la culture populaire a honte d'ellemême. L'anthropologue ou le théoricien de gauche qui voudraient parler en son nom ne sont pas ses représentants. Mais souvent il n'y a pas d'autres représentants.

Même si on connaissait les revendications réelles du peuple, beaucoup de ces revendications sont trop disparates ou incompatibles, impossibles à satisfaire dans la mesure où le peuple n'a ni les habitudes ni les institutions qui permettent de dégager des compromis politiques et des objectifs viables. Je me souviens d'un Amérindien qui réclamait un retour du Canada à l'état précolombien. Il voulait un retour à la chasse et à la cueillette. Mais il ne voulait pas voir que la population du Canada devrait se réduire de beaucoup pour vivre selon ces techniques. Son intransigeance était à la mesure de ses frustrations et de son impuissance. ${ }^{1}$

Quand il s'agit de développement international et d'aide internationale, un débat et un vote démocratiques sont pratiquement impossibles dans la mesure où les décisions, et les coûts et bénéfices qui s'ensuivent, ne relèvent pas d'une seule collectivité politique. Des propos raisonnables sont d'autant plus difficiles à tenir et à faire prévaloir, que les différents intérêts en jeu n'ont pas des moyens comparables pour soutenir un argument. Il n'y a ni langage ni critères communs pour un débat commun. Il n'y a même pas de procédure assurant une voix à toutes les parties concernées. Entre ces dernières, la disparité et les inégalités sont immenses.

Sur cette toile de fond, je propose quelques considérations très partielles qui, je l'espère, apparaîtront raisonnables et pertinentes pour un débat sur les fins de l'aide au développement international, au moins à l'intérieur de ce pays.

Je partirai du contraste entre l'utilitarisme et une théorie fondée sur les droits de la personne. "The Fundamental point of contrast, and conflict, between utilitarian and right-based views is

1. En fait, certains peuples uutochtones du Canada ont appris à négocier habilement avec les gouvernements provinciaux ou fédéral. Ils se sont donné les moyens politiques pour se concerter et gagner de l'influence. Ils savent comment mettre le bon droit de leur côté ou exploiter la mauvaise conscience de l'opinion des "blancs». 
that the former, at least in their basic theory, aggregate the interests or preferences of all the persons of parties who are being taken into account, whereas the latter insist, to the end, on the separateness of persons. For the utilitarian, the ultimate determination and the ultimate justification of every moral requirements lies in its relations - perhaps a complex and indirect relation to something that represents the pooling of all individual purposes. The rights-theorist takes it to be morally important to respect and protect - at all levels, not at some only - the separate interests of each individual in a way that goes beyond the pool. » (J.L. Mackie, «Rights, Utility and Universalisation » in Utility and Rights, R.G. Frey, editor, University of Minnesota Press, 1984, p. 86).

L'utilitarisme vise à maximiser les satisfactions d'un groupe sur une certaine période. Cette perspective est séduisante. D'abord, elle se prête au calcul, ce qui passe pour une preuve de rationalité. Ensuite, elle envisage les satisfactions non d'un individu isolé, à un moment donné, mais d'un ensemble historique. Elle ne souffre donc pas d'étroitesse. Enfin, cette perspective exclut les jugements de valeur : c'est là l'objet de choix individuels, ce sont ces choix qui déterminent les utilités que l'utilitarisme prend en compte et prétend maximiser. Mais une telle perspective appelle des correctifs. En effet, peut-on, pour obtenir un bénéfice social plus grand, imposer des coûts inacceptables à certains individus? La plus grande somme de satisfactions nettes pour un groupe ne justifie pas n'importe quel sacrifice ni une répartition inégale des sacrifices au sein du groupe. La maximisation des satisfactions futures ne justifient pas n'importe quel sacrifice actuel, même si ce sont les mêmes personnes qui souffrent aujourd'hui et bénéficieront de satisfactions plus tard. On parle des droits de la personne et de théorie morale fondée sur de tels droits, pour, entre autres raisons, corriger l'indifférence de l'utilitarisme au sort des individus actuels. Les philosophes spécialisés en éthique ont discuté abondamment de ces questions. A Theory of Justice de John Rawls (Harvard University Press, 1971) est probablement l'œuvre majeure en la matière.

En passant en revue les objectifs de l'aide au développement, tels qu'ils sont explicités par les plus hautes instances dans un pays comme le Canada, on trouve de manière répétée l'expression suivante : aider les plus démunis à s'aider eux-mêmes. En fait, cette 
proposition, qui semble limpide, recèle une difficulté sur laquelle je voudrais réfléchir. Elle est justement à la croisée d'un souci utilitariste et d'un souci au sujet des droits.

Aider les plus pauvres, c'est se soucier de leurs droits comme de leurs besoins. On peut insister davantage sur les uns ou sur les autres. ${ }^{2}$ Si l'on dit que l'aide internationale doit avoir pour but la protection des droits fondamentaux ou des droits de la personne, c'est que ce sont les droits des moins bien nantis qui sont les moins assurés et que l'on veut assurer davantage. Mais pour intervenir en faveur des plus pauvres, on prendra pour cible des pays, des régions, des groupes ou des secteurs défavorisés. On privilégiera le développement des ressources humaines, des mesures sanitaires ou des mesures en faveur de l'éducation de base. On tentera d'améliorer les conditions économiques et sociales pour qu'il soit possible de respecter les droits fondamentaux des plus démunis. On ne s'intéressera donc pas seulement aux plus démunis ou aux droits fondamentaux. En effet, ni les uns ni les autres ne correspondent à un secteur isolé sur lequel on pourrait agir isolément. Par ailleurs, pour aider les plus démunis à s'aider eux-mêmes, pour rendre leur développement autonome, il faut favoriser des dynamismes sociaux, économiques et politiques, attirer et canaliser des investissements, encourager des innovations, éduquer des techniciens et des cadres, aider des entreprises viables plutôt que celles qui n'ont pas d'avenir. Mais alors l'aide peut fort bien s'adresser à d'autres que les plus démunis. En fait, les plus démunis sont généralement difficiles à rejoindre si ce n'est indirectement.

Un pays, une région, un secteur de la société ou un groupe ne sont évidemment pas homogènes : les individus qui en font partie ne sont pas également défavorisés. D'autre part, pour aider une collectivité, il convient de provoquer en son sein l'émergence de savoir-faire et d'organisations. Cela ne la rend pas nécessairement plus égalitaire, du moins pas à court terme. Bref, pour aider efficacement des individus à s'aider eux-mêmes, il faut poursuivre des objectifs collectifs et adopter un point de vue que je qualifierais

2. J'évite de parler ici des besoins, de la comparaison entre besoins et droits, de la comparaison entre les avantages d'un langage basé sur les besoins et les avantages d'un langage basé sur les droits. Sur les besoins, voir Meeting Needs, par David BAYBroOKs, Princeton University Press, 1987. 
d'utilitariste. Mais le respect des droits de la personne doit demeurer une priorité à deux égards : ce respect est le but ou l'un des buts ultimes qui justifient des objectifs collectifs; d'autre part, il permet de rectifier les effets inacceptables d'une politique définie d'un point de vue collectif et utilitariste. Voilà qui demande quelques précisions.

L'utilitarisme demeure probablement le paradigme selon lequel on juge d'une politique à première vue. Il permet de mesurer son efficacité, en ce sens qu'il permet de comparer ses bénéfices globaux et ses coûts globaux. Mais il ne permet pas, comme on l'a déjà dit, de juger de la répartition des bénéfices et des coûts. En fait, il ne permet pas non plus d'apprécier le bien-fondé des bénéfices et des coûts, c'est-à-dire des préférences individuelles au regard desquelles les bénéfices et les coûts, les utilités gagnées et les utilités perdues sont évalués. En effet, pour juger des utilités, il prétend pouvoir les comparer d'un point de vue intersubjectif, il prétend pouvoir réduire les préférences des différents sujets à un commun dénominateur. Les choix subjectifs au sujet des utilités ne sont pris en compte qu'à la condition d'être ceux d'un sujet type. Si on croit pouvoir éviter cette difficulté en s'en remettant au libre jeu du marché, c'est qu'on ne voit pas combien ce jeu n'a pas la liberté qui permettrait de résoudre la difficulté. Premièrement, l'offre conditionne très souvent la demande. Deuxièmement, le pouvoir d'achat n'est pas également réparti, la demande des acteurs économiques n'est donc pas vraiment concurrentielle, les uns peuvent satisfaire des besoins superflus et d'autres ne peuvent satisfaire des besoins essentiels. L'offre est donc fonction des demandes monnayables et non des besoins de chacun.

Envisager les droits de la personne, c'est adopter un point de vue qui, sans faire de jugement de valeur indu à la place d'autrui, permet de corriger certaines insuffisances de l'utilitarisme au sens où je l'entends ici. ${ }^{3}$ Évidemment, il convient d'entendre les droits de la personne au sens large. Et le premier d'entre eux, c'est le droit de s'exprimer politiquement et de s'associer pour revendiquer des

\footnotetext{
3. On pourrait me reprocher de ne pas mentionner ici les principes de Rawls. Ils sont et plus précis et plus conformes à ma conception de la justice que les formulations que j'emploie ici. Mais mon point de départ est une situation donnée où s'affrontent deux conceptions auxquelles correspondent mes formulations. Mon but est d'éclairer ces conceptions et leur opposition dans une situation donnée, non de disserter sur la justice.
} 
droits civils, politiques, économiques et sociaux. Un tel droit est respecté là "où les pauvres s'organisent pour transiger avec leur gouvernement, où les gouvernements encouragent les pauvres à mettre des organisations sur pied pour les représenter et où les gouvernements sont disposés à traiter avec ces organisations» (Quidoit en profiter. Rapport surles politiques et programmes $d u$ Canada en matière d'aide publique au développement. Comité permanent des Affaires étrangères et du commerce extérieur, Chambre des communes, 1987, p. 77).

Parler des droits, c'est se mettre au point de vue de l'individu et des garanties institutionnelles qui lui sont reconnues par une société. Ce n'est certes pas le seul point de vue à partir duquel on peut gérer une société. Une entreprise, une équipe sportive, une Église rassemblent des individus pour d'autres fins qu'une distribution des droits et obligations réciproques de ces individus. ${ }^{4} \mathrm{Mais}$ une société politique, tel un État, devrait avoir comme justification et fin ultime, ou comme une de ses justifications et une de ses fins, le respect et la promotion de ses membres et de leurs droits. Et sans attendre d'avoir atteint cette fin ultime, les gestionnaires d'une telle société se doivent, en tout temps, de respecter et de faire respecter un minimum de droits fondamentaux. Ceux-ci peuvent être définis de manière différente. Des droits économiques et sociaux peuvent s'ajouter et s'accroître avec la prospérité collective. Mais un minimum de droits doit être garanti aux individus 5 dès aujourd'hui, même si on rêve de faire mieux à l'avenir. Ce sera une manière de protéger les individus contre des politiques qui les sacrifieraient au bien collectif ou à l'avenir de tous.

On me dira probablement que j'adopte ici un point de vue libéral, ethnocentrique, que jaccorde trop d'importance aux droits individuels et pas assez au bien commun ou aux valeurs singulières des cultures qui nous sont étrangères. C'est vrai. Il y a un danger certain à ne parler que des droits de chacun sans considérer le bien de tous, à ne parler que des droits formels sans se soucier de la

4. C'est ce que j'ai renté de démontrer dans «Dire le droit n'est pas le faire» (Dialogue XXVI-2, 1987) en me basant sur l'article de Lon L. FULLER, "The Forms and Limits of Adjudication ", Harvard Law Review', n' 921, 1978.

5. Je voudrais ajouter « et aux minorités». Je ne peux malheureusement pas m'étendre ici sur les droits collectifs. Ils devraient compléter les droits de la personne et les concrétiser plutôt que s'y opposer. Mais il y a là bien des difficultés conceptuelles et pratiques. 
réalité économique et sociale. Le discours officiel des États-Unis au sujet des droits de la personne dans les pays qui échappent à leur emprise, est manifestement borné, unilatéral et idéologique. Il illustre bien ce danger. Mais contre les aveuglements d'une technocratie ou d'un parti de Dieu, de la droite ou de la gauche, il n'y a pas d'autres remparts que le pluralisme garanti par les institutions et que l'insistance sur les droits des citoyens.

Sans doute, cette insistance est-elle d'origine occidentale. Mais elle peut offrir un moyen de se défendre contre de nouvelles manières de gérer les sociétés, contre l'accroissement des interdépendances, contre les rationalités bureaucratique et économique qui, à partir de l'Occident, s'étendent inéluctablement, renversant solidarités et valeurs traditionnelles. Pour illustrer mon propos, je ne retiendrai qu'un exemple: la planification.

Elle est devenue un instrument courant dans les administrations publiques et privées. Elle emprunte à la conception utilitariste : il s'agit de maximiser des bénéfices, d'éviter des manques à gagner, de tirer le meilleur parti possible de possibilités et de ressources limitées. Cela implique une manière de voir les choses qui ne va pas sans oillères. Une manière de voir que ne corrigent plus des traditions morales et des solidarités coutumières, car la planification s'est souvent établie sur leurs ruines.

Je recense quatre cillères. Premièrement, on l'a déjà vu, la planification a tendance à ignorer le problème de la distribution des coûts et des bénéfices. Deuxièmement, elle risque de négliger les coûts et les bénéfices qui ne s'évaluent pas en termes budgétaires. Et ceux qui s'évaluent dans ces termes sont souvent évalués en fonction de prix courants qui ne sont pas nécessairement la meilleure mesure de leur valeur, même économique à long terme. Troisièmement, des objectifs qui ne sont pas des variables ayant des effets sur d'autres variables risquent d'être ignorés. Ainsi l'éducation de la main-d'œuvre future et sa santé sont non seulement désirables en soi, elles sont aussi des facteurs qui se renforcent l'un l'autre et servent l'économie nationale. Les conséquences d'une politique de la santé en faveur des jeunes et les conséquences d'une politique d'éducation ont des effets multiples. C'est ce qui justifie ces politiques plus facilement que des allocations en faveur des vieillards. Quand on n'envisage l'utilité d'un objectif que dans la 
série de ses conséquences, comment ne pas sous-estimer tous ceux qui ne peuvent être appréciés en fonction de leurs répercussions sur l'avenir? Enfin, et cette objection n'est pas la moindre, la prétention même d'une rationalisation générale se heurte au fait que nous ne savons pas tout ce qui nous importe. Il y a des valeurs qui sont déjà vécues avec intensité sans être encore reconnues. Comment en tenir compte dans une planification?

Ce ne sont pas là des raisons suffisantes pour renoncer à l'idéal d'une rationalisation plus poussée des décisions, mais ce sont des raisons pour considérer toute planification générale comme devant respecter les droits fondamentaux de chacun, d'une part, comme devant faire l'objet d'un débat et d'une révision constants, d'autre part. Elle ne devrait pas être une affaire des seuls technocrates, une manière de dissimuler des choix de classe sous le couvert de la rationalité administrative ou économique. L'élaboration d'un plan devrait être l'occasion de mettre cartes sur table au sujet de la distribution des coûts et des bénéfices, un stimulant pour la discussion démocratique.

C'est sans doute trop attendre, surtout dans des pays en voie de développement où l'opinion publique n'est pas trop informée. Mais c'est aussi la raison pour laquelle le respect des droits de la personne peut jouer un rôle stratégique dans ces pays. Ils ne sont pas un luxe mais une manière d'assurer que les plus pauvres ne seront pas oubliés et auront aussi un mot à dire dans le débat au sujet du développement, au sujet du prix à donner aux choses, aux hommes, à leurs sacrifices et à leurs besoins. Dans cette perspective, il faut surtout insister sur le droit, pour les plus démunis, de s'organiser pour traiter avec le gouvernement et les administrations.

Dans le cours de ce texte, j'ai parlé des droits de la personne selon trois points de vue différents et ce ne sont pas tout à fait les mêmes droits qui étaient invoqués quand on changeait de point de vue. Il vient d'être question du droit de s'associer et de s'exprimer politiquement. Ce n'est manifestement là qu'un des droits de la personne et, par ailleurs, la participation politique des citoyens n'est pas seulement une affaire de droit. Il fut aussi question des droits de la personne au pluriel: garanties pour les individus ${ }^{6}$,

6 . "et pour les minorirés", devrait-on ajouter. 
instituées par la loi, exigibles devant les tribunaux. Ces droits sont censés protéger les individus contre l'arbitraire, notamment contre l'arbitraire de l'État. Ils peuvent aussi servir à protéger les individus contre des sacrifices qu'on prétendrait leur imposer, au nom même du bien collectif ou de droits à venir. Plus haut, il fut question également de la promotion des droits de la personne comme du but ou d'un des buts ultimes de l'action politique et de l'aide au développement. Dans ce contexte, la notion de droit de la personne prend une extension maximale et sort du champ juridique.

Défendre les droits de l'homme, au sens large ou au sens étroit, c'est sans doute adopter un point de vue occidental mais c'est aussi donner des moyens, et notamment un langage en passe de devenir universel, à tous et chacun, pour se défendre, au milieu des bouleversements des mœurs traditionnelles, contre la rationalité économique, la raison des États et des bureaucraties, les croisades en faveur d'un salut national ou de lendemains qui chantent.

\section{REMARQUE FINALE}

Au terme de ce texte, j'ai la nette impression de m'être borné à quelques aspects des problèmes soulevés. Pourtant je voudrais consacrer ces dernières pages à un tout autre problème : la perspective que le philosophe pourrait adopter et devrait adopter, me semble$\mathrm{t}$-il, vis-à-vis des questions complexes que pose la pratique sociale, en général, et des questions qui forment le titre de ce texte, en particulier.

Le public attend parfois du philosophe qu'il sorte des ornières académiques et ose tracer des perspectives morales et politiques, fondées sur un savoir spécialisé. Or, comme n'importe quel expert, le philosophe se réfugie volontiers derrière les frontières étroites de son expertise pour ne pas se compromettre. Il préfère ne pas se mêler de trop près aux complications de la pratique. D'ailleurs, quand il s'en mêle, il lui arrive de proférer des bêtises monumentales. Il risque de devenir un propagandiste politique ou bien de croire aux mirages d'un meilleur des mondes. J'estime pourtant qu'il est possible de suivre une voie plus fructueuse sans tomber dans ces facilités. Je ne suggère aucunement que je fus capable de suivre cette voie et c'est pourquoi je voudrais en suggérer le tracé. 
À titre d'exemple, je voudrais rapporter l'expérience d'un ethnologue, A.F. Robertson, chargé, avec d'autres, d'un projet en vue d'améliorer l'économie et l'écologie du lac Dal de Srînagar, au Cachemire (A.F. Robertson, «The Dal Lake: Reflections on an Anthropological Consultancy in Kashmir » in Anthropology Today III-2, 1987). Une équipe multidisciplinaire avait été invitée par le gouvernement indien pour ce projet. On y retrouvait des experts en tous genres. Mais Robertson découvrit rapidement qu'il n'avait aucune expertise particulière. Il écouta donc les gens vivant du lac, sur le lac, autour du lac. Il écouta les agronomes, les économistes, les spécialistes du traitement de l'eau, tous ces experts avec qui il faisait équipe. Il écouta les représentants du gouvernement de New Dehli, du gouvernement de l'État de Jammu et Cachemire, de la municipalité de Srînagar. Il finit par recommander que l'on traite les eaux usées des grands hôtels et des égouts de Srînagar. Mais il recommanda aussi qu'on laisse les cultures maraîchères et les « houseboats » sur le lac car, n'en déplaise aux écologistes purs et durs, cela fait l'attrait touristique du lac et permet à quantité de pauvres gens de vivre des touristes ou de leur potager, sans causer de dommages significatifs à la qualité de l'eau.

De telles recommandations n'ont pas la clarté unilatérale qu'offre un discours d'expert, car elles tiennent compte de points de vue multiples et souvent contradictoires, de tous les résidents, de plusieurs experts et de divers groupes d'intérêt. Ces recommandations sont le résultat sans éclat d'un compromis prudent.

Quand il est consulté sur des questions pratiques, le philosophe lui aussi se doit de demeurer ouvert aux multiples facettes de la réalité, sensible aux besoins des hommes concrets et aux valeurs dont ils vivent. Il n'est pas suffisant d'être sensible aux droits de la personne hors de toute autre considération, mais reconnaître ces droits, c'est commencer à rendre justice à chacun, préférer tenir compte de la pluralité des projets et des perspectives plutôt que d'imposer la sienne.

Le philosophe ne doit pas se laisser obnubiler par les recettes de gauche ou de droite, de tel ou tel prêcheur, de tel ou tel spécialiste à la mode. C'est là toute sa sagesse et elle ne relève pas vraiment d'une expertise particulière. D'une part, il lui faut reprendre les perspectives de diverses expertises, non seulement 
pour tirer au clair leurs propos, limites ou préjugés, mais aussi pour comprendre les hommes et les enjeux sociaux selon différents points de vue, pour ensuite souligner les responsabilités morales et politiques qui découlent de ce savoir. Si le philosophe refuse de se laisser enfermer dans un savoir spécialisé, c'est parce qu'il a le souci du concret et de la pratique. D'autre part, il doit se méfier des apôtres et des croyants de tous poils, mais il lui faut aussi les écouter, demeurer pieusement attentif aux valeurs silencieuses comme à ceiles qui se proclament. C'est pour être attentif à leur diversité qu'il doit d'abord se méfier des enthousiasmes unilatéraux, ne pas accepter les solutions toutes faites mais apprécier différentes manières d'apprécier les choses.

Certes, ces tâches sont celles de tout homme. Elles ne relèvent pas d'une profession particulière. Le philosophe le sait mieux que tout autre. Mais il peut meitre une certaine passion à demeurer froid, analytique et curieux, une certaine réserve à sympathiser avec les passions, les valeurs et les mentalités dont il est témoin. C'est ainsi que je conçois sa profession. C'est ainsi qu'il peut faire bon usage de son expertise particulière et de celles des autres.

Département de philosopbie

Collège de Saint-Laurent 